\title{
Tree diversity, stand structure and community composition in tropical forest of Rajaji tiger reserve, Northern India
}

\author{
Akash* \\ Department of Botany and Microbiology, Gurukula Kangri University; Haridwar-249401 \\ (Uttarakhand), India \\ Navneet \\ Department of Botany and Microbiology, Gurukula Kangri University; Haridwar-249401, \\ (Uttarakhand), India

\section{B.S. Bhandari} \\ Ecology Laboratory, Department of Botany and Microbiology, H.N.B. Garhwal University, \\ Srinagar Gharwal-246174 (Uttarakhand), India \\ ${ }^{*}$ Corresponding author. E-mail: Saklanibotany@gmail.com

\begin{abstract}
In present study, we present data on tree diversity, stand structures and community composition in six sites of tropical forest in Rajaji tiger reserve, Northern India. The enumeration of 72 plots results a total of 19,050 individuals, 47 species, 42 genera, 25 families in which Holoptelia integrifolea, Dalbergia sissoo, Shorea robusta, Cassia fistula and Trewia nudiflora were the species which showed higher importance value index (IVI) in the study area. The stand density of the six sites ranges from $149.99-397.91$ hac- $^{1}$ where as the total basal area of trees ranges from $3.612-46.813 \mathrm{~m}^{2} / \mathrm{hac}^{-}{ }^{1}$. The Shannon diversity index ranged from 1.35 to 2.51 , Simpson index ranged from $0.097-0.446$, Margalef index ranged $2.584-4.9$, The Evenness index ranged from $0.551-0.852$ in the study area. Further the studied area has showed ample evidences from indices in supporting the higher floristic diversity and stand structure after providing the present area as a status of tiger reserve.
\end{abstract}

Keywords: Density, Diversity, Tree species, Tropical forest, Tree species

\section{Article Info}

DOI:10.31018/jans.v10i3.1791

Received: June 9, 2018

Revised: July 30, 2018

Accepted: August 3, 2018

\section{How to Cite}

Akash, et al. (2018).

Tree diversity, stand structure and community composition in tropical forest of Rajaji tiger reserve, Northern India. Journal of Applied and Natural Science, 10(3): 945 - 953

\section{INTRODUCTION}

Tropical forests are regarded one of the most biological rich habitat on earth and have been recognized to harbour a significant proportion of world biodiversity (Myers et al 2000; Baraloto et al., 2013). These forests provide significant ecological services such as conservation of the habitats of plants and animals, prevention of soil erosion and species conservation as well (Armenteras et al 2009). Many factors like seedling survivorship, seed quality and recruitment are the important factors which are playing significant contribution in the maintaining the tree composition of tropical forests (Connell, 1971).

Tropical forest accounts for approximately $86 \%$ of the total forest cover in India (Singh and Singh, 1988) and the dry tropical forest accounts for approximately $38.2 \%$ of the forest cover but unfortunately the dry deciduous forests are being converted into dry savannah, scrub and dry grasslands (Champion and Seth, 1968; Singh and Singh, 1989). These forest are degrading at a rate of $3.5 \%$ (Puyravaud and Davidar, 2010). The main reason behind for the loss of tropical forest are invasive species, fragmentation, deforestation, over-exploitation as well as the climate change. (Gardner et al., 2009; Morris, 2010; Anonymous, 2013). The degradation of these tropical forest is likely to continue in the future (Bradshaw et al., 2009). So this condition is calls for in depth study of the demographic stability of the species in these tropical forest (Sagar and Singh, 2003). The tropical moist deciduous forests are found throughout India except in the north-western and western regions of the country. The tropical moist deciduous forests receive a range of rainfall between $100-200 \mathrm{~cm}$. In the higher ranges of northeastern India and the hilly areas of Uttarakhand, West Bengal, these forests mainly comprises of $S$. robusta, D. sissoo, Tectona grandis, Emblica officinalis, Bombex ceiba, Schlifera oleosa etc. Uttarakhand has about 34,359 hectares of total forest cover and approximately $63 \%$ of total area of the state. This state is $358 \mathrm{~km}$ in length and $322 \mathrm{~km}$ in breadth. The Haridwar forest division cover 7304.60 hactare of forest cover in Uttarakhand state. Our objective is to study the stand structure, 
community composition and diversity of tree species in tropical forest of Rajjai tiger reserve.

\section{MATERIALS AND METHODS}

The study was conducted in six different forest sites of Haridwar-Pauri forest division of Rajaji tiger reserve. The tiger reserve is an essentials part of the terai landscape between Sharda and Yamuna river in Shivalik landscape (Akash et al., 2018a). The study area comes division under Chilla forest range. The sub tropical forest of tiger reserve comes under Shivalik hill. Rajai Tiger reserve is located in northern India at $29^{\circ} 51^{\prime} \mathrm{N}$ to $30^{\circ} 15^{\prime} \mathrm{N}, 077^{\circ} 52^{\prime} \mathrm{E}$ to $078^{\circ} 22^{\prime} \mathrm{E}$ at an elevations from $250-1,100$ above mean sea level. It falls within the Gangetic Plains biogeographic zone and upper Gangetic Plains province (Rodgers et al. 2002). Shivalik hill is categorized as part of the Indo-Gangetic Plains and has great significance in India's biogeography due to intermingling of flora from the Indo Malayan and Palaearctic regions (Sivakumar et al. 2010). The Chilla range of the reserve is one of the great centre of attractions for tourists (Akash et al., 2018b)

Assessment of tree species composition, stand structure and distribution along the elevation gradient was done to cover the six sites of the study area. The six sites area given below along with elevation gradient and coordinates which are taken from the GPS Instruments (Table 1 and Fig. 1). Sampling and data analysis: The six sites were studied to cover the stand structure, diversity and distribution of the species in the studied area. At each site, 12 Quadrates (a total of 72 Quadrates) of $20^{*} 20 \mathrm{~m}^{2}$ were randomly laid down to observe the tree species of the area. To determine the population structure of the forest, trees were measured for cbh with a girthing tape. The representative taxa were collected and after that identified with the help of regional floras (Raizada and Saxena, 1978; Gaur, 1999; Duthie, 1903-29) and prepared into herbarium. The voucher specimens were submitted in the Department of Botany and Microbiology, Gurukul Kangri University, India.

Data analysis: The individuals recorded in the discrete plot samples, vegetation data were analyzed quantitatively for relative density, relative frequency, relative dominance and basal area. The importance value index (IVI) of tree species was calculated as the summation of relative frequency, relative density, and relative dominance (Curtis and Mclntosh 1950). After that structural composition was analyzed by comparing the distribution of tree diameter classes. The data were also used to interpret community indices like species diversity $\left(\mathrm{H}^{\prime}\right)$ of different tree species and was calculated by using the Shannon-Weiner Index (Shannon and Weiner 1963)

The Shannon weaver index, Simpson index, Species richness, (Margalef index) and evenness (Whittaker index) were calculated for each community. These all diversity indices were calculated with the help of following formulas:

$\mathrm{H}^{\prime}=-\Sigma$ pi In $\mathrm{p}_{\mathrm{i}}$ (Shannon and Wiever, 1963)..Eq.1 Where, $\mathrm{pi}=\mathrm{ni} / \mathrm{N}$, which denotes the importance probability of each species in a population; ni = importance value for species "i", $\mathrm{N}=$ total of importance values.

$\mathrm{Cd}=\Sigma \mathrm{p}_{\mathrm{i}}{ }^{2} \quad$ (Simpson, 1949).... Eq.2

Where $\mathrm{Cd}=$ Concentration of dominance

$\mathrm{R} \quad=\mathrm{S}-1 / \mathrm{ln}(\mathrm{N}) \quad$ (Margalef, 1968)......Eq.3

Where $S=$ Number of species, $N=$ Total number of Individuals

Statistical analysis: The correlation analysis was used to determine the relation between various phytoscociological attributes (Prasad, 2011) as mentioned in Tables 2-6, Table 8 and Figs.2-3

\section{RESULTS}

Floristic structure and Species composition: A total of 19,050 individuals of trees belonging to the 47 species among 42 genera and 25 families from 72 quadrates of $20 \times 20 \mathrm{~m}^{2}$ were enumerated in tropical forest of Northern India.

Stand density and diversity: Trees stand density ranged between $4775 \mathrm{ind} / \mathrm{hac}$ of GS to $1825 \mathrm{ind} /$ hac in KS. The other sites showed moderate density of tree in the study area. In LS the density of trees was 3275 ind/ hac, 2225 ind/hac in KR whereas in KB- 6-8 it was 3825 ind/hac and 3125 ind/hac in SF. The total basal area was highest recorded in KB- 6-8 (46.81 $\left.\mathrm{m}^{2} \mathrm{hac}^{-1}\right)$ whereas the

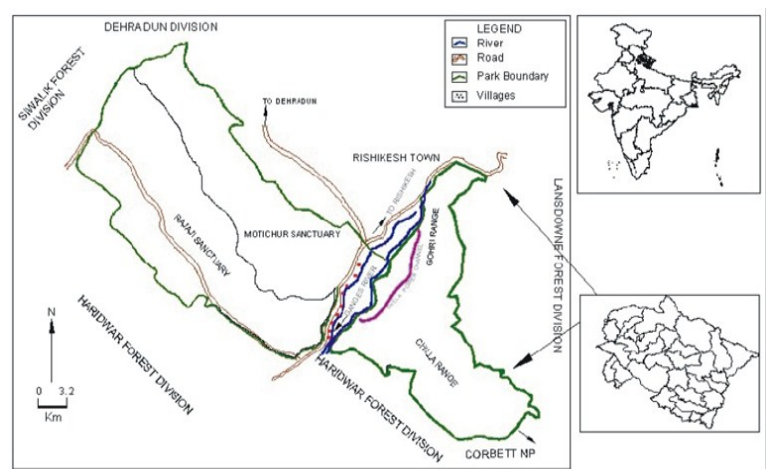

Fig. 1. Map of the Rajaji tiger reserve (Modified from (Rasily, 2008).

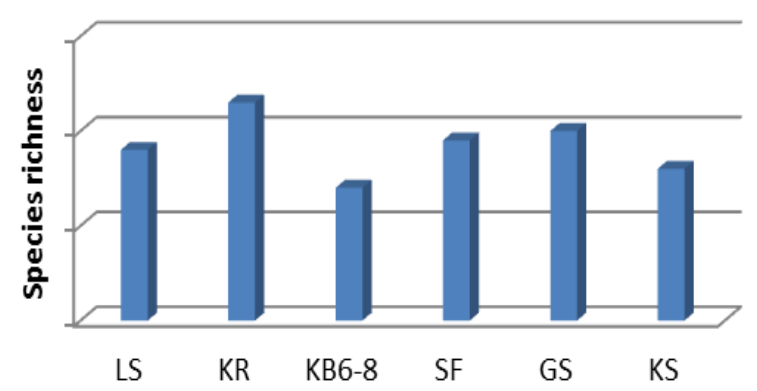

Fig. 2. Species richness in different studied sites $(L S=$ Lalsroth, $K R=$ Kodiya ridge, $K B=$ Kodiyabelt 6 $8, S F=$ Sofuti, GS= Ghasiram, KS=Kharasroth). 
Table 1. Coordinates analysis of the study sites.

\begin{tabular}{llll}
\hline Sites & Elevation & Latitude(N) & Longitute(E) \\
\hline 1-Lalsroath & $378.6 \mathrm{~m}$ & $30^{\circ} 00^{\prime} 35.9^{\prime \prime} \mathrm{N}$ & $78^{\circ} 16^{\prime} 21.8 \mathrm{E}$ \\
2-Kodiya ridge & $350 \mathrm{~m}$ & $30^{\circ} 0^{\prime} 35.8^{\prime \prime} \mathrm{N}$ & $78^{\circ} 15^{\prime} 45.7 \mathrm{E}$ \\
3-Kodiyabelt 6-8 & $458.1 \mathrm{~m}$ & $29^{\circ} 59^{\prime} 41.0^{\prime} \mathrm{N}$ & $78^{\circ} 17^{\prime} 41.1 \mathrm{E}$ \\
4-Sofuti & $482.7 \mathrm{~m}$ & $29^{\circ} 59^{\prime} 28.8^{\prime}{ }^{\prime} \mathrm{N}$ & $78^{\circ} 17^{\prime} 44.2 \mathrm{E}$ \\
5-Ghasiram sroath & $304 \mathrm{~m}$ & $29^{\circ} 57^{\prime} 44.92^{\prime \prime} \mathrm{N}$ & $78^{\circ} 11^{\prime} 33.81 \mathrm{E}$ \\
6- Kharasroath & $318 \mathrm{~m}$ & $29^{\circ} 56^{\prime} 51.9^{\prime \prime} \mathrm{N}$ & $78^{\circ} 10^{\prime} 46.17 \mathrm{E}$ \\
\hline
\end{tabular}

Tabel 2. List of tree species encountered in Lalsroth with their family names, densities $\left(\right.$ hac- $\left.{ }^{1}\right)$, basal area $\left(\mathrm{m}^{2}\right.$ hac- ${ }^{1}$ ), Relative values and importance value Index.

\begin{tabular}{llllllll}
\hline Species & Family & Density/hac & Basal area & RF & RD & RDo & IVI \\
\hline Holopotelia integrifolea & Ulmaceae & 160.4 & 14.08 & 25.532 & 61.105 & 70.239 & 156.87 \\
Cedrella toona & Meliaceae & 2.082 & 0.662 & 2.128 & 0.795 & 3.306 & 6.229 \\
Alstonia scholaris & Apocynaceae & 2.082 & 0.134 & 2.128 & 0.795 & 0.67 & 3.593 \\
Naringi crenulata & Rutaceae & 6.25 & 0.1194 & 4.255 & 2.386 & 0.596 & 7.237 \\
Cassia fistula & Fabaceae & 8.35 & 0.209 & 6.383 & 3.179 & 1.046 & 10.608 \\
Mallotus phillipensis & Euphorbiaceae & 31.25 & 0.521 & 17.022 & 11.932 & 2.602 & 31.556 \\
Ehretia laevis & Ehertiaceae & 10.4 & 0.261 & 6.383 & 3.971 & 1.305 & 11.659 \\
Oroxylum indicum & Bignoniaceae & 4.165 & 0.059 & 4.255 & 1.59 & 0.297 & 6.142 \\
Listea chinensis & Lauraceae & 10.25 & 0.15 & 6.383 & 3.914 & 0.748 & 11.045 \\
Terminalia bellerica & Combretaceae & 4.165 & 0.855 & 2.128 & 1.59 & 4.264 & 7.982 \\
Ziziphus oenoplia & Rhamnaceae & 2.082 & 0.0169 & 2.128 & 0.795 & 0.085 & 3.008 \\
Aegle marmelos & Rutaceae & 2.082 & 0.026 & 2.128 & 0.795 & 0.132 & 3.055 \\
Crateva relegiosa & Capparaceae & 8.32 & 0.114 & 8.511 & 3.179 & 0.569 & 12.259 \\
Syzygium cumini & Myrtaceae & 2.08 & 0.02 & 2.128 & 0.795 & 0.132 & 3.055 \\
Ficus religiose & Moraceae & 2.08 & 0.106 & 2.128 & 0.795 & 0.529 & 3.452 \\
Adina cordifolia & Rubiaceae & 2.08 & 2.652 & 2.128 & 0.795 & 13.277 & 16.2 \\
Morus alba & Moraceae & 2.08 & 0.02 & 2.128 & 0.795 & 0.1 & 3.023 \\
Celtis australis & Cannabaceae & 2.08 & 0.033 & 2.128 & 0.795 & 0.0167 & 2.939 \\
Total & & 261.91 & 20.05 & 100 & 100 & 99.99 & 299.91 \\
\hline
\end{tabular}

Tabel 3. List of tree species encountered in Kodiya ridge with their family names, densities $\left(\right.$ hac $^{-1}$ ), basal area $\left(\mathrm{m}^{2}\right.$ hac- $\left.^{1}\right)$, Relative values and importance value Index.

\begin{tabular}{llllllll}
\hline Species & Family & $\begin{array}{l}\text { Densityl } \\
\text { hac }\end{array}$ & $\begin{array}{l}\text { Basal } \\
\text { area }\end{array}$ & RF & RD & Rdo & IVI \\
\hline Dalbergia sissoo & Fabaceae & 87.5 & 1.18 & 24.001 & 47.193 & 32.678 & 103.872 \\
Trewia nudiflora & Euphorbiaceae & 10.41 & 0.2545 & 8 & 5.618 & 7.048 & 20.666 \\
Ehertia leavis & Ehertiaceae & 10.41 & 0.32 & 8 & 5.618 & 8.882 & 22.5 \\
Ziziphus oenoplia & Rhamnaceae & 6.25 & 0.161 & 2 & 3.371 & 4.476 & 9.847 \\
Chordia dichotoma & Boraginaceae & 2.08 & 0.069 & 2 & 1.124 & 0.47 & 3.594 \\
Emblica officinalis & Fabaceae & 10.41 & 0.151 & 6 & 5.618 & 4.204 & 15.822 \\
Mallotus phillipensis & Euphorbiaceae & 14.5 & 0.187 & 10 & 7.865 & 5.18 & 23.04 \\
Holoptelia integrifolia & Ulmaceae & 2.08 & 0.018 & 2 & 1.124 & 0.5 & 3.624 \\
Butea monosperma & Fabaceae & 2.08 & 0.035 & 2 & 1.124 & 0.972 & 4.096 \\
Psidium gujava & Myrtaceae & 2.08 & 0.037 & 2 & 1.124 & 1.025 & 4.149 \\
Ficus glomerata & Moraceae & 2.08 & 0.0597 & 2 & 1.124 & 1.653 & 4.777 \\
Cedella toona & Meliaceae & 2.08 & 0.0175 & 2 & 1.124 & 0.485 & 3.609 \\
Legerestroemia indica & Lythraceae & 8.33 & 0.209 & 6 & 4.494 & 5.811 & 16.305 \\
Cassia fistula & Fabaceae & 2.08 & 0.0597 & 2 & 1.124 & 1.653 & 4.777 \\
Ficus religiose & Moraceae & 2.08 & 0.0812 & 2 & 1.124 & 2.25 & 5.374 \\
Acacia spp & Fabaceae & 2.08 & 0.0335 & 2 & 1.124 & 0.93 & 4.054 \\
Melia azadirachta & Meliaceae & 2.08 & 0.0335 & 2 & 1.124 & 0.93 & 4.054 \\
Acacia catatue & Fabaceae & 2.08 & 0.01833 & 4 & 1.124 & 0.507 & 5.631 \\
Pterospermum acirifolium & Malvaceae & 2.08 & 0.02031 & 2 & 1.124 & 0.562 & 3.686 \\
Caliandra haematocephala & Fabaceae & 2.08 & 0.0265 & 2 & 1.124 & 0.734 & 3.858 \\
Bombex ceiba & Bombecaceae & 6.25 & 0.1825 & 4 & 3.37 & 5.052 & 12.422 \\
Bahunia variegata & Fabaceae & 2.08 & 0.0812 & 2 & 1.124 & 2.25 & 5.374 \\
Adina cordifolia & Rubiaceae & 2.08 & 0.424 & 2 & 1.124 & 11.752 & 14.876 \\
Total & & $\mathbf{1 8 5 . 4}$ & $\mathbf{3 . 6 1 2}$ & $\mathbf{1 0 0}$ & $\mathbf{1 0 0}$ & $\mathbf{1 0 0}$ & $\mathbf{3 0 0}$ \\
\hline
\end{tabular}

lowest in $\mathrm{KR}\left(3.61 \mathrm{~m}^{2} \mathrm{hac}^{-1}\right)$. The other sites showed moderate range of basal area.

Diversity of tree species in the studied plots of the area was calculated by using the Shannon-Weiner

diversity index $\left(\mathrm{H}^{\prime}\right)$. The Shannon-Weiner diversity index showed that the highest diversity was in $\mathrm{KR}$ (2.161) and the lowest diversity was in KB (1.350), with dominance of Simpson's value ranging from 
Akash, et al. / J. Appl. \& Nat. Sci. 10 (3): 945 - 953 (2018)

Tabel 4. List of tree species encountered in Kodiyabelt 6-8 with their family names, densities(hac- $\left.{ }^{1}\right)$, basal area $\left(\mathrm{m}^{2}\right.$ hac- $\left.^{1}\right)$, Relative values and importance value Index.

\begin{tabular}{llllllll}
\hline Species & Family & Density/hac & Basal area & RF & RD & Rdo & IVI \\
\hline Shorea robusta & Dipterocarpaceae & 208.25 & 43.649 & 20.692 & 65.424 & 93.241 & 179.357 \\
Mallotus phillipensis & Euphorbiaceae & 41.65 & 0.606 & 20.692 & 13.085 & 1.295 & 35.072 \\
Aegle marmelos & Rutaceae & 6.25 & 0.1037 & 12.069 & 1.964 & 0.222 & 14.255 \\
Listea chinensis & Lauraceae & 10.25 & 0.119 & 10.346 & 3.222 & 0.254 & 13.82 \\
Naringi crenulata & Rutaceae & 10.25 & 0.18 & 8.62 & 3.22 & 0.386 & 12.226 \\
Cassia fistula & Fabaceae & 6.25 & 0.267 & 5.173 & 1.964 & 0.571 & 7.708 \\
Legestroemia parviflora & Lythraceae & 2.083 & 0.016 & 1.724 & 0.654 & 0.036 & 2.416 \\
Ficus religiose & Moraceae & 6.25 & 0.902 & 3.444 & 1.964 & 1.928 & 7.339 \\
Holoptelia integrifolea & Ulmaceae & 2.08 & 0.134 & 1.724 & 0.654 & 0.287 & 2.665 \\
Erythrina indica & Fabaceae & 2.08 & 0.186 & 3.447 & 0.654 & 0.398 & 4.499 \\
Crateva relegiosa & Capparaceae & 2.08 & 0.0414 & 1.724 & 0.654 & 0.088 & 2.466 \\
Schlifera oleosa & Sapindaceae & 2.08 & 0.0595 & 1.724 & 0.654 & 0.127 & 2.505 \\
Miliusa velutina & Annonaceae & 2.08 & 0.0699 & 3.447 & 0.654 & 0.149 & 4.25 \\
Ehretia laevis & Ehertiaceae & 16.66 & 0.476 & 5.173 & 5.234 & 1.018 & 11.425 \\
Total & & $\mathbf{3 1 8 . 3 0 8}$ & $\mathbf{4 6 . 8 1 3}$ & $\mathbf{1 0 0}$ & $\mathbf{9 9 . 9 9 9}$ & $\mathbf{1 0 0}$ & $\mathbf{2 9 9 . 9 9}$ \\
\hline
\end{tabular}

Tabel 5. List of tree species encountered in Sofuti with their family names, densities(hac- ${ }^{1}$ ) basal area $\left(\mathrm{m}^{2}\right.$ hac- $\left.^{1}\right)$, Relative values and importance value Index.

\begin{tabular}{llllllll}
\hline Species & Family & Density/hac & Basal area & RF & RD & Rdo & IVI \\
\hline Mallotus phillipensis & Euphorbiaceae & 41.666 & 1.036 & 12.069 & 16 & 12.057 & 40.126 \\
Listea chinensis & Lauraceae & 10.414 & 0.084 & 5.173 & 4 & 0.987 & 10.16 \\
Naringi crenulata & Rutaceae & 39.583 & 0.083 & 10.345 & 15.2 & 9.654 & 35.199 \\
Holoptelia integrifolea & Ulmaceae & 20.833 & 0.605 & 3.448 & 8 & 7.039 & 18.487 \\
Ehertia leavis & Ehertiaceae & 4.166 & 0.04 & 3.448 & 1.6 & 0.47 & 5.518 \\
Cassia fistula & Fabaceae & 47.016 & 0.983 & 13.792 & 18.4 & 11.441 & 43.633 \\
Schlifera oleosa & Sapindaceae & 6.25 & 0.531 & 3.448 & 2.4 & 6.179 & 12.027 \\
Anogeissus latifolia & Combretaceae & 2.083 & 0.0812 & 1.724 & 0.8 & 0.945 & 3.469 \\
Shorea robusta & Dipterocarpaceae & 2.083 & 0.1666 & 1.724 & 0.8 & 1.938 & 4.462 \\
Erythrina suberosa & Fabaceae & 6.25 & 0.756 & 5.173 & 2.4 & 8.802 & 16.375 \\
Aegle marmelos & Rutaceae & 16.666 & 0.228 & 8.621 & 6.4 & 2.657 & 17.678 \\
Adina cordifolia & Rubiaceae & 8.333 & 2.516 & 6.897 & 3.2 & 29.269 & 39.366 \\
Emblica officinalis & Fabaceae & 2.083 & 0.02 & 1.724 & 0.8 & 0.236 & 2.76 \\
Butea monosperma & Fabaceae & 6.25 & 0.0731 & 1.724 & 2.4 & 0.851 & 4.975 \\
Gewia asiatica & Tiliaceae & 8.33 & 0.0996 & 5.173 & 3.2 & 1.158 & 9.531 \\
Erythrina indica & Fabaceae & 10.41 & 0.142 & 3.448 & 4 & 1.654 & 9.102 \\
Mitrgyana parviflora & Rubiaceae & 6.25 & 0.1375 & 3.448 & 2.4 & 1.532 & 7.38 \\
Bauhinia purpurea & Fabaceae & 8.333 & 0.1366 & 5.173 & 3.2 & 1.589 & 9.531 \\
Chordia dichotoma & Boraginaceae & 12.5 & 0.132 & 3.448 & 4.8 & 1.541 & 9.789 \\
Total & & 260.41 & 8.598 & 100 & 100 & 99.99 & 299.57 \\
\hline
\end{tabular}

Tabel 6. List of tree species encountered in Ghasiram sroath with their family names, densities(hac- ${ }^{1}$ ) basal area $\left(\mathrm{m}^{2}\right.$ hac- $\left.{ }^{-1}\right)$, Relative values and importance value Index.

\begin{tabular}{llllllll}
\hline Species & Family & Density/hac & Basal area & RF & RD & Rdo & IVI \\
\hline Trewia nudiflora & Euphorbiaceae & 260.416 & 9.598 & 25.532 & 65.446 & 50.788 & 141.766 \\
Naringi crenulata & Rutaceae & 2.083 & 0.018 & 2.128 & 0.523 & 0.096 & 2.747 \\
Listea chinensis & Lauraceae & 25 & 0.0432 & 6.383 & 6.283 & 0.229 & 12.895 \\
Ficus benghalensis & Moraceae & 2.083 & 0.7313 & 2.128 & 0.523 & 3.87 & 6.521 \\
Mallotus phillipensis & Euphorbiaceae & 31.25 & 0.557 & 10.639 & 7.853 & 2.95 & 21.442 \\
Azadirachta indica & Meliaceae & 2.083 & 0.02031 & 2.128 & 0.523 & 0.107 & 2.758 \\
Ziziphus mauritiana & Rhamnaceae & 2.083 & 1.2089 & 2.128 & 0.523 & 6.397 & 9.048 \\
Crateva religiosa & Capparaceae & 4.1667 & 0.0339 & 4.255 & 1.047 & 0.18 & 5.482 \\
Mitragyna parviflora & Rubiaceae & 2.083 & 0.4245 & 2.128 & 0.523 & 2.246 & 4.897 \\
Careya arborea & Lecythidaceae & 4.1667 & 1.074 & 4.255 & 1.047 & 5.687 & 10.989 \\
Ficus auriculata & Moraceae & 2.083 & 0.5799 & 2.128 & 0.523 & 3.069 & 5.72 \\
Bahunia variegata & Fabaceae & 12.5 & 0.6237 & 8.511 & 3.141 & 3.3 & 14.952 \\
Syzium cumini & Myrtaceae & 18.75 & 0.4335 & 6.383 & 4.712 & 2.294 & 13.389 \\
Holoptelia integrifoea & Ulmaceae & 14.583 & 0.753 & 6.383 & 3.665 & 3.988 & 14.036 \\
Albizia lebbek & Fabaceae & 2.083 & 0.0414 & 2.128 & 0.523 & 0.219 & 2.87 \\
Adina cordifolia & Rubiaceae & 2.083 & 0.2388 & 2.128 & 0.523 & 1.264 & 3.915 \\
Cassia fistula & Fabaceae & 2.083 & 0.018 & 2.128 & 0.523 & 0.096 & 2.747 \\
Acacia catatue & Fabaceae & 2.083 & 0.1239 & 2.128 & 0.523 & 0.656 & 3.307 \\
Putranjiva roxburghii & Euphorbiaceae & 2.083 & 0.7594 & 2.128 & 0.523 & 4.018 & 6.669 \\
Melia azadirachta & Meliaceae & 4.1667 & 1.625 & 4.255 & 1.047 & 8.598 & 13.9 \\
Total & & $\mathbf{3 9 7 . 9 1 2 3}$ & $\mathbf{1 8 . 9}$ & $\mathbf{1 0 0}$ & $\mathbf{9 9 . 9 9}$ & $\mathbf{1 0 0}$ & $\mathbf{3 0 0}$ \\
\hline \hline
\end{tabular}


Akash, et al. / J. Appl. \& Nat. Sci. 10 (3): 945 - 953 (2018)

Tabel 7. List of tree species encountered in Kharsroath with their family names, densities(hac- $\left.{ }^{1}\right)$ basal area $\left(\mathrm{m}^{2}\right.$ hac- ${ }^{1}$ ), Relative values and importance value Index.

\begin{tabular}{llllllll}
\hline Species & Family & Density/hac & Basal area & RF & RD & Rdo & IVI \\
\hline Holoptelia integrifolea & Ulmaceae & 60.416 & 1.4511 & 26.316 & 40.278 & 27.05 & 93.644 \\
Schlifera oleosa & Sapindaceae & 4.1667 & 0.8492 & 5.263 & 2.778 & 15.831 & 23.872 \\
Mallotus phillipensis & Euphorbiaceae & 31.25 & 0.65 & 15.79 & 20.834 & 12.133 & 48.757 \\
Putranjiva roxburghii & Euphorbiaceae & 4.1667 & 0.162 & 5.263 & 2.778 & 3.029 & 11.07 \\
Thevetia peruviana & Apocynaceae & 4.1667 & 0.0372 & 2.631 & 2.778 & 0.694 & 6.103 \\
Ficus palmata & Moraceae & 2.0832 & 0.02 & 5.263 & 1.389 & 0.379 & 7.031 \\
Delonix regia & Fabaceae & 2.0832 & 0.0933 & 2.631 & 1.389 & 1.739 & 5.759 \\
Cassia fistula & Fabaceae & 2.0832 & 0.02798 & 2.631 & 1.389 & 5.217 & 9.237 \\
Grewia asiatica & Tiliaceae & 6.25 & 0.0731 & 2.631 & 4.167 & 1.363 & 8.161 \\
Acacia catatue & Fabaceae & 4.166 & 0.0466 & 2.631 & 2.778 & 0.87 & 6.279 \\
Listea chinensis & Lauraceae & 8.333 & 0.0835 & 10.526 & 5.556 & 1.558 & 17.64 \\
Adina cordifolia & Lauraceae & 2.083 & 1.209 & 2.631 & 1.389 & 22.539 & 26.559 \\
Ehertia leavis & Ehertiaceae & 6.25 & 0.0633 & 7.895 & 4.167 & 1.18 & 13.242 \\
Cedrella toona & Meliaceae & 2.083 & 0.1061 & 2.631 & 1.389 & 1.979 & 5.999 \\
Crateva relegiosa & Capparaceae & 8.333 & 0.0722 & 2.631 & 5.556 & 1.347 & 9.534 \\
Melia azadirachta & Meliaceae & 2.083 & 0.16585 & 2.631 & 1.389 & 3.092 & 7.112 \\
Total & & 149.997 & 5.3645 & 99.99 & 100 & 100 & 299.99 \\
\hline
\end{tabular}

Table 8. Floristic richness, density (hac-1), total basal cover $\left(\mathrm{m}^{2 /} \mathrm{hac}^{-}\right)$and diversity indices of six sites in tropical forest Rajaji tiger reserve, Northern India.

\begin{tabular}{lllllll}
\hline Variables & LS & KR & KB 6-8 & SF & GS & KS \\
\hline No. of Genera & 18 & 21 & 14 & 19 & 19 & 16 \\
No. of Family & 16 & 13 & 12 & 12 & 12 & 11 \\
No. of Species & 18 & 23 & 14 & 19 & 20 & 16 \\
Density/hac & 261.91 & 185.4 & 318.308 & 260.41 & 397.912 & 149.997 \\
Total basal area $\left(\mathrm{m}^{2 /}\right.$ hac $\left.^{-1}\right)$ & 20.05 & 3.6121 & 46.813 & 8.598 & 18.9 & 5.3645 \\
Shannon Index & 1.593 & 2.161 & 1.35 & 2.51 & 1.459 & 2.059 \\
Simpson index & 0.389 & 0.235 & 0.446 & 0.097 & 0.44 & 0.203 \\
Margalef index & 3.51 & 4.9 & 2.584 & 3.728 & 3.617 & 3.496 \\
Eveness & 0.551 & 0.689 & 0.511 & 0.689 & 0.511 & 0.852 \\
\hline
\end{tabular}

$\mathrm{LS}=$ Lalsroth, $\mathrm{KR}=$ Kodiya ridge, $\mathrm{KB}=$ Kodiyabelt 6-8, SF= Sofuti, GS= Ghasiram, KS=Kharasroth

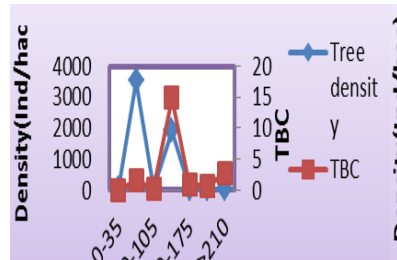

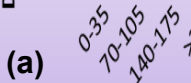
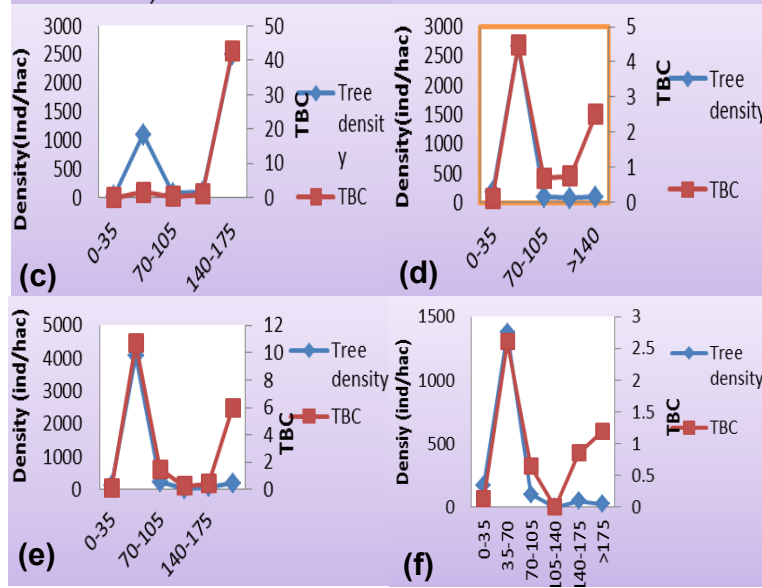

Fig.3 (a-f). Contribution of tree Stand density and total basal cover based in different girth class (in $\mathrm{Cm}$ ) of all the sites (a). - Lalsroath, (b).- Kodiya ridge, (c).Kodiyabelt 6-8, (d).-Sofuti, (e). Ghasiram sroath (f). Kharasroath.
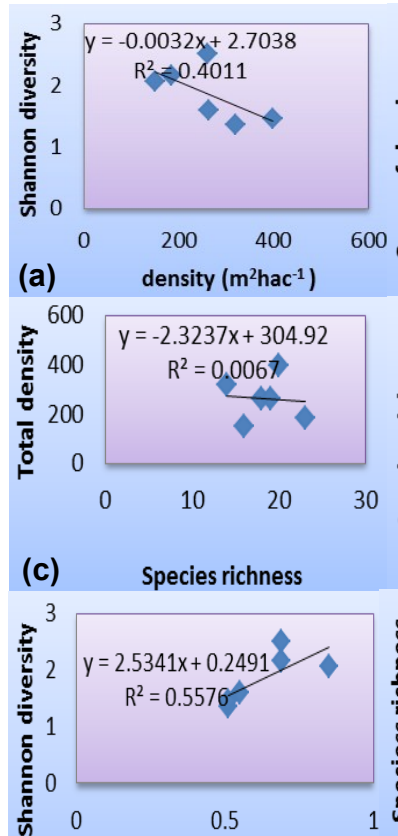

(e)

Evenness

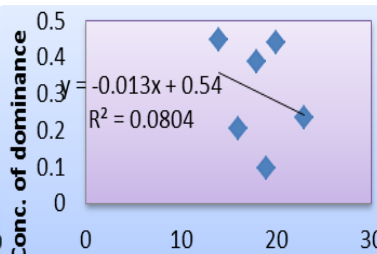

(b) Species richness

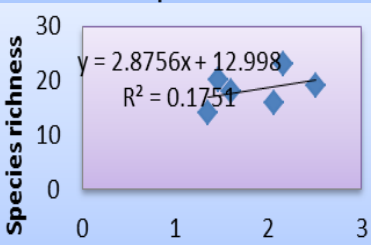

(d) Shannon diversity

Fig. 4 (a-f): Correlation among various phytosociological attributes. 
0.097 (SF)- 0.440 (GS). The Margalef species richness index was highest recorded in KR (4.90) and lowest was recorded in KB (2.584). The evenness index was highest recorded in KS (0.852) and lowest was in GS, KB which was 0.511(Table 8).

The sites represented different dominant and codominant species. In LS, Holoptelia integrifolea (IVI= 156.876) was the most dominant species followed by other co-dominant species Mallotus phillipensis (IVI= 31.556) and Crateva relegiosa $(\mathrm{IVI}=12.259)$ and so on (Table 7). On the other hand, in KR, Dalbergia sissoo (103.872) was the most dominant followed by other co-dominant species like Trewia nudiflora (IVI=20.666), Legerestroemia indica (IVI= 16.305) and others (Table 8). In KB 6-8, Shorea robusta (IVI= 179.357) was the most dominant species followed by the co-dominant species like Mallotus phillipensis (IVI= 35.072), Naringi crenulata (IVI=13.82) and others (Table 9). In SF, Cassia fistula (IVI= 43.633) was the most dominant species followed by species Mallotus phillipensis $(\mathrm{IVI}=40.126)$ and other whereas in GS, Trewia nudiflora (IVI=141.766) was most dominant species followed by Mallotus phillipensis (IVI $=21.442$ ) and other but in GS, Holoptelia integrifolea ( IVI= 93.644) was most dominant tree species followed by Mallotus phillipensis (IVI= 48.757), Adina cordifolia (IVI $=2126.559)$ and others (Tables 2- 7).

Correlation analysis: The correlation between different phytosociological parameter are given in Fig. 4 (a-f). The density (hac ${ }^{-1}$ ) was negatively correlated with the Shannon diversity $(r=-0.633)$ and species richness $(-0.081)$. The Shannon diversity was positively correlated with species richness $(r=0.418)$ whereas the species richness was negatively correlated with concentration of dominance $(r=-0.28)$ and but positively correlated with Evenness $(r=0.066)$. On the other hand Evenness was positively correlated with the Shannon diversity $(r=0.746)$.

\section{DISCUSSION}

Tropical forests are rich in floral species density and diversity (Richards 1952; Paijmans 1970) but many factors affect their diversity (Janzen 1970; Connell 1971; Hubbell 1979; Parthasarathy 1999). The overall structural pattern of the forest community revealed the habitat is dominated by Shorea robusta, Holoptelia integrifolea, Dalbergia sissoo, Mallotus phillipensis, Cassia fistula and codominated with Ziziphus oenophlia, Z. mauritiana, Ehretia laevis, Naringi crenulata etc. These dominant species in stand sometimes restrict the light availability to other species of main canopy and ground flora in the undisturbed and mildly disturbed stands.

The absolute total stand density $\left(\mathrm{hac}^{-1}\right)$ of the six sites ranged from $149.99 /$ hac $^{-1}-397.91$ hac $^{-1}$, in which highest density was recorded in GS and lowest was in KR. The recorded density values of our study did not vary considerably from values reported by other authors of Garhwal Himalaya (Singhal and Soni 1989 form Mussoorie forest division, Adhikari and Tiwari, 1991) but the total basal area in our study was more or less similar to the study of other workers in different tropical forest of Northern India. The total basal area $\left(\mathrm{m}^{2} /\right.$ hac) of trees ranged from $3.612(\mathrm{KR})-46.813 \mathrm{~m}^{2}$ / ha (KB 6-8). These values are comparable to the value reported by Raturi, 2012 as 3.18- $43.62 \mathrm{~m}^{2} /$ hac from Rudraprayagh forest division, Lata and Bisht 1991 as $13.60-71.25 \mathrm{~m}^{2} / \mathrm{hac}$ from moist temperate forest of Pauri, Bhat, 2012 as 2.91-37.96 $\mathrm{m}^{2} /$ hac form Kedarnath Wildlife Santuary in Northern India.

The Shannon weaver diversity index of the tree species in present study area varies from 1.35 (KB, 6-8) to 2.51 (SF). These values are comparable with those reported by Ghidiyal et al., 1998 as 1.86-2.73 from Garhwal forest division, Raturi et al., 2012 as $0.78-3.45$ from Rudraprayagh forest division, Singh et al., 2014 as 0.66-2.69 from Gorakhal forest of Nainital, Pant and Samant, 2012 as $0.74-2.66$ from Khokan Wildlife Santuary, Uniyal et al., 2010 as 0.70-3.08 from Devalgarh forest division in the Uttarakhand Himalaya. The dominance of forest sometimes increased with an increase in the elevation. Simpson's index value (con. of dominance) was 0.097 in SF $(482.7 \mathrm{~m}$ ) which was highest at the elevation gradient. This situation might be due to tolerance-based dominance of only few of the tree species under severe environmental conditions. According to Baduni and Sharma (1999), the concentration of dominance is always affected by the first three relatively important species in a community. The concentration of dominance $(\mathrm{Cd})$ in our study ranged from 0.097 (SF) -0.446 (KB 6-8). These result are almost similar to the study carried out by Raturi, (2012) in subtropical forest of Rudraprayagh district in Garhwal Himalaya whose results value ranged from $0.09-0.63$. All the results on vegetation composition and phytosociological were in accordance with the earlier findings in tropical forests of Bhagirathi basin in Garhwal region (Sharma et al. 2009, 2014, 2015)

Every species play significant role and there is a definite quantitative relationship between abundant and rare species (Bhandari et al., 1999). High importance value index ((IVI)) indicates that all the available resources are being utilized by that species and left over being trapped by other competitors and associated species. In present study area, high importance value was observed for $H$. integrifolea ( in LS- 156.876), D. sissoo ( in KR-103.872), S. robusta (in- KB6-8- 179.357), C. fistula (in SF- 43.633), T. nudifolra (in GS- 
141.766), $H$. integrifolea (in KR- 93.644). Similar results were also observed by Raturi (2012) in Rudraprayagh forest of Garhwal Himalaya for Quarcus lecuotrichophora as the dominant species and by Kukshal.et al., 2009 for Callipedium parviflorum in Badiyargarh forerst of Garhwal Himalaya.

Rajji tiger reserve is characterize by sub tropical climate, varied topography with rich alluvial soil and fragile ecosystem which makes it one of the most diverse area of Northern India. These factors have evolved new association of forest community inside the reserve. In present study, high value of $H$. integrifloea, S. robusta, D. sissoo, C.fistula and $T$. nudiflora revels that all the source being utilized by these species in each community as compared to the other associated species. High importance value and basal cover of these species reveals that dominancy over other species in a community. The dominancy of these species also reveals that these species in each community are highly utilizing the entire sources.

Highest basal area of in KB 6-8 and highest diversity in SF inside the tiger reserve reveals that it is providing conservation to major forest of S. robusta and various mixed forest communities in Northern India.

\section{Conclusion}

Rajaji tiger reserve cover the huge part of northern India and providing many goods and ecosystem services which are essential for improving and maintaining the livelihoods of humans, animals and plants in addition to performing various ecological functions. The five relative highest ecological importance (IVI) species in the six sites of study area were $H$. integrifolea, $D$. sissoo, S. robusta, $C$. fistula and T. nudiflora. The results also revealed more than twenty species showed the unstable population hence hampered regeneration but diversity and stand structure increases significantly due to the its conservation status and strict provision for community in past years. Further study suggests that the studied area is still in a recovery stage. Since it had faced severe anthropogenic pressure prior to the establishment of the exclosure with the fence plants, sociological characters such as frequency, density, abundance as well as the basal area were being influenced by anthropogenic as well as natural stress in past, but now various protection strategies like restriction to Gujjar community, fire line, eradication of harmful weed, Gujjar rehabilitation programme further support species regeneration inside the tiger reserve.

\section{ACKNOWLEDGEMENTS}

The authors are grateful to the Director and Forest guards of the Rajaji tiger reserve for their help during the field work.

\section{REFERENCES}

1. Anonymous, (2013). Managing Forest Resources for Sustainable Development: An Evaluation of World Bank Group Experience. IEG, World Bank, Washington, USA.

2. Adhikari, R.S and Tiwari A. (1991) Microbial decomposition of oak leaf litter in temperate forest of Kumaun Himalayas. Acta Bot Indica, 19:195-200.

3. Akash,. Navneet and Bhandari, B.S (2018a). Phytosociological studies, biodiversity conservation in a sub tropical moist deciduous forest of Rajaji Tiger reserve; Uttarakhand, India. International Journal of Research and Analytical Reviews 5 (3): 39-51.

4. Akash,. Navneet and Bhandari, B.S (2018b). Phytosociological investigation, Biodiversity conservation and Life form pattern in a Holeptelia integrifolia community under Rajaji Tiger Reserve, Uttarakhand, India. International Research Journal of Biological Sciences, 7(7):1-8.

5. Armenteras, D., Rodríguez, N., and Retana, J. (2009). Are conservation strategies effective in avoiding the deforestation of the Colombian Guyana Shield?. Biological Conservation, 142(7), 1411-1419.

6. Baduni, N. P., and Sharma, C. M. (1999). Community structure and growing stock variation in Quercus floribunda forest on different aspects of Garhwal Himalaya. Bangladesh Journal of Forest Science, 28, 82-93.

7. Baraloto, C., Molto, Q., Rabaud, S., Hérault, B., Valencia, R., Blanc, L. and Thompson, J. (2013). Rapid simultaneous estimation of aboveground biomass and tree diversity across Neotropical forests: a comparison of field inventory methods. Biotropica, 45(3), 288-298.

8. Bhandari, B.S., Mehta, J.P. Nautiyal, B.P. and Tiwari, S.C. (1997). Structure of a Chir- Pine community along an altitudinal gradient in Garhwal Himalaya. International journal of Ecology and Environmental Sciences, 23:67-74.

9. Bhat, J. A. (2012). Diversity of flora along an altitudinal gradient in Kedarnath Wildlife Sanctuary. Doctoral dissertation, Ph.D. Thesis HNB Garhwal University, Srinagar Uttarakhand, India

10.Bradshaw, C. J., Sodhi, N. S., and Brook, B. W. (2009). Tropical turmoil: a biodiversity tragedy in progress. Frontiers in Ecology and the Environment, 7(2), 7987.

11.Champion, S. H., and Seth, S. K. (1968). A revised survey of the forest types of India. New Delhi.

12.Connell, J.H. (1971). On the role of enemies in preventing competitive exclusion in some marine animals and in rain forest trees. In: den Boer, P.J, Gradwell, G.R(ed). Dynamics of populations. Wageningen, The Netherlands: Centre for Agricultural Publication and Documentation. Pp. 298e312.

13.Curtis, J. T., and McIntosh, R. P. (1950). The interrelations of certain analytic and synthetic phytosociological characters. Ecology, 31(3), 434-455.

14.Duthie, J.F. (1903). Flora of the Upper Gangetic Plain and the adjacent Shiwalik and Sub Himalayan tracts. Vol. 3,Superitendent of government Printing, Calcutta.

15.Gardner, T. A., Barlow, J., Chazdon, R., Ewers, R. M., Harvey, C. A., Peres, C. A., and Sodhi, N. S. (2009). Prospects for tropical forest biodiversity in a human-modified world. Ecolog Letters, 12(6), 561- 
582

16.Gaur, R.D. (1999). Flora of District Garhwal, Northwest Himalaya: with ethnobotanical notes. Transmedia Publishing, Srinagar, Garhwal, Uttarakhand, India.

17.Ghildiyal, S., Baduni, N. P., Khanduri, V. P., and Sharma, C. M. (1998). Community structure and composition of oak forests along altitudinal gradient in Garhwal Himalaya. Indian Journal of Forestry, 21 (3), 242-247.

18.Gogate, M.G, Kumar, A. (1993) An ecological audit of teak plantations in west Chandrapur Project Division. Ind For 119:265-294

19. Hartshorn, G.S. (1980). Neotropical forest dynamics. Biotropica 12: 23-30

20.Hubbell, S. P. (1979). Tree dispersion, abundance, and diversity in a tropical dry forest. Science, 203 (4387), 1299-1309.

21.Jaccard, P. (1912). The distribution of the flora in the alpine zone. New phytologist, 11(2): 37-50.

22.Janzen, D. H. (1970). Herbivores and the number of tree species in tropical forests. The American Naturalist, 104(940), 501-528

23.Jha, C.S. and Singh J.S. (1990). Composition and dynamics of dry tropical forest in relation to soil texture. Journal of Vegetational Science, 1: 609-614.

24.Joshi, N.K. and Tiwari, S.C.(1990). Phytosociological analysis of woody vegetation along an altitudinal gradient in Garhwal. Indian Journal of forestry, 13:322-328.

25.Joshi, R. (2016). Mammalian fauna of rajaji national park, india: a review on ecological observations and checklist. Check List, 12(3): 1892.

26.Kukshal, S., Nautiyal, B. P., Anthwal, A., Sharma, A., and Bhatt, A. B. (2009). Phytosociological investigation and life form pattern of grazinglands under pine canopy in temperate zone, Northwest Himalaya, India. Research Journal of Botany, 4(2): 55-69.

27.Lata, K., and Bisht, N. S. (1991). Quantitative analysis and regeneration potential of moist temperature forest in Garhwal Himalayas. Indian Journal of forestry, 14(2): 98-106.

28.Laurance, W. F., Oliveira, A. A., Laurance, S. G., Condit, R., Nascimento, H. E., Sanchez-Thorin, A. C. and Dick, C. W. (2004). Pervasive alteration of tree communities in undisturbed Amazonian forests. Nature, 428(6979), 171.

29.Morris, R. J. (2010). Anthropogenic impacts on tropical forest biodiversity: a network structure and ecosystem functioning perspective. Philosophical Transactions of the Royal Society B: Biological Sciences, 365(1558), 3709-3718.

30.Margalef R. (1968). Perspectives in Ecological Theory. Chicago: University of Chicago Press.

31.Myers, N., Mittermeier, R. A., Mittermeier, C. G., Da Fonseca, G. A., and Kent, J. (2000). Biodiversity hotspots for conservation priorities. Nature, 403 (6772), 853.

32.Odum, E.P. (1971). Fundamentals of ecology. $3^{\text {rd }}$ edition. W.B. Saunders Co., Philadelphia. XXIV, 574.

33.Pant, S., and Samant, S. S. (2012). Diversity and regeneration status of tree species in Khokhan Wildlife Sanctuary, north-western Himalaya. Tropical Ecology, 53(3), 317-331.

34.Pande, P.K., Negi, J.D.S. and Sharma. S.C. (1996). Plant species diversity and vegetation analysis in moist temperate Himalayan forest. Proceeding of
Abstract First Indian Ecological Congress, New Delhi, 27-31.

35.Paijmans, K. (1970). An analysis of four tropical rain forest sites in New Guinea. The Journal of Ecology, 77-101.

36.Pausas, J. G., and Austin, M. P. (2001). Patterns of plant species richness in relation to different environments: an appraisal. Journal of Vegetation Science, 12(2), 153-166.

37.Parthasarathy, N. (1999). Tree diversity and distribution in undisturbed and human-impacted sites of tropical wet evergreen forest in southern Western Ghats, India. Biodiversity and Conservation, 8(10), 13651381.

38.Prasad, S. (2011). Fundamentals of Biostatistics, Emkay publishers, Puyravaud JP, Davidar P, Laurance WF. Cryptic loss of India's native forests. Conservation Letters 2010: 3(6):390-394.

39.Raizada, M.B. and Saxena, H. (1978). Flora of Mussorie. Bishen Singh, Mahendra Pal Singh, Dehradun. Rasily SS..Management plane of Rajaji tiger reserve, Uttarakhand, India. 2008.

40.Raturi, G. P. (2012). Forest community structure along an altitudinal gradient of district Rudraprayag of Garhwal Himalaya, Indian Ecologia, 2(3): 76-84.

41.Richards D.W. 1952. The Tropical Rainforest. Cambridge University Press, Cambridge, UK.

42.Rodgers, W. A., Panwar, H. S., and Mathur, V. B. (2002). Wildlife Protected Area Network in India: A Review (Executive Summary), Wildlife Institute of India. Dehradun. 44p.

43.Sagar R. and Singh J.S. (2003). Predominant phenotypic traits of disturbed tropical dry deciduous forest vegetation in northern India. Community Ecology, 4 (1): 63-71.

44.Sagar, R., and Singh, J. S. (2005). Structure, diversity, and regeneration of tropical dry deciduous forest of northern India. Biodiversity and Conservation, 14 (4), 935-959.

45.Saxena, A. K., and Singh, J. S. (1982). A phytosociological analysis of woody species in forest communities of a part of Kumaun Himalaya. Plant Ecology, 50 (1): 3-22.

46.Sharma, C. M., Suyal, S., Gairola, S., and Ghildiyal, S. K. (2009). Species richness and diversity along an altitudinal gradient in moist temperate forest of Garhwal Himalaya. Journal of American Science, 5 (5), 119-128.

47.Sharma, C. M., Mishra, A. K., Prakash, O., Dimri, S., and Baluni, P. (2014). Assessment of forest structure and woody plant regeneration on ridge tops at upper Bhagirathi basin in Garhwal Himalaya. Tropical Plant Research, 1(3), 62-71.

48.Sharma, C. M., Mishra, A. K., Krishan, R., Tiwari, O. P., and Rana, Y. S. (2016). Variation in vegetation composition, biomass production, and carbon storage in ridge top forests of high mountains of Garhwal Himalaya. Journal of Sustainable Forestry, 35(2), 119 $-132$.

49.Shannon, C.E. and Weaver, W. (1963). The Mathematical theory of communication. University of Illinois Press, Urbana.

50.Simpson, E. H. (1949). Measurement of diversity. Nature, 163(4148), 688.

51.Singh, K. P., and Singh, J. S. (1988). Certain structural and functional aspects of dry tropical forest and savan- 
Akash, et al. / J. Appl. \& Nat. Sci. 10 (3): 945 - 953 (2018)

na. International Journal of Ecology and Environmental Sciences. 14: 31-45

52.Singh, J.S., Singh, K.P and Agrawal, M. (1991) Environmental degradation of the Obra-RenukootSingrauli area, India, and its impact on natural and derived ecosystems. Environ, 11:171-180

53.Singhal, R. M., and Soni, S. (1989). Quantitative ecological analysis of some woody species of Mussoorie Himalayas (UP). Indian Forester, 115(5), 327336.

54.Singh V.P. and Singh J.S. (1989). Man and forests: a case study from the dry tropics of India. Environmental Conservation 16: 129-136.

55.Sivakumar, K., Sathyakumar, S., and Rawat, G. S. (2010). A preliminary review on conservation status of Shivalik landscape in Northwest, India. Indian Forester, 136(10), 1376.

56.Sork, V. L., Davis, F. W., Smouse, P. E., Apsit, V. J., Dyer, R. J., Fernandez-M, J. F., and Kuhn, B. (2002). Pollen movement in declining populations of California Valley oak, Quercus lobata: where have all the fathers gone?. Molecular Ecology, 11(9), 1657-1668.

57.Uniyal, P., Pokhriyal, P., Dasgupta, S., Bhatt, D., and Todaria, N. P. (2010). Plant diversity in two forest types along the disturbance gradient in Dewalgarh Watershed, Garhwal Himalaya. Current Science, 938 $-943$

58.Whitford, P. B. (1949). Distribution of woodland plants in relation to succession and clonal growth. Ecology, 30(2): 199-208. 